$$
\begin{array}{llll}
\lrcorner & \lrcorner
\end{array}
$$

Log minimal models according to Shokurov

Caucher Birkar

\lrcorner $」$

\rfloor

象 


\title{
Log minimal models according to Shokurov
}

\author{
Caucher Birkar
}

\begin{abstract}
Following Shokurov's ideas, we give a short proof of the following klt version of his result: termination of terminal log flips in dimension $d$ implies that any klt pair of dimension $d$ has a log minimal model or a Mori fibre space. Thus, in particular, any klt pair of dimension 4 has a log minimal model or a Mori fibre space.
\end{abstract}

\section{Introduction}

All the varieties in this paper are assumed to be over an algebraically closed field $k$ of characteristic zero. We refer the reader to Section 2 for notation and terminology.

The following conjecture is perhaps the most important problem in birational geometry.

Conjecture 1.1 (Minimal model). Let $(X / Z, B)$ be a Kawamata log terminal (klt) pair. Then it has a log minimal model or a Mori fibre space.

The 2-dimensional case of this conjecture is considered to be classical. The 3-dimensional case was settled in the 80's and 90's by the efforts of many mathematicians, in particular Mori, Shokurov and Kawamata. The higher-dimensional case has seen considerable progress in recent years, thanks primarily to Shokurov's work on the existence of log flips, which paved the way for further progress. The conjecture is also settled for pairs of general type [Birkar et al. 2006], and inductive arguments have been proposed for pairs of nonnegative Kodaira dimension [Birkar 2007]. For a more detailed account of the known cases of this conjecture, see the introduction to [Birkar 2007].

Shokurov [2006] proved that the log minimal model program (LMMP) in dimension $d-1$ and termination of terminal log flips in dimension $d$ imply Conjecture 1.1 in dimension $d$ even for log canonical (lc) pairs. (In this paper, by termination of terminal $\log$ flips in dimension $d$, we will mean termination of any sequence $X_{i} \rightarrow X_{i+1} / Z_{i}$ of $\log$ flips $/ Z$ starting with a $d$-dimensional klt pair $(X / Z, B)$ which is terminal in codimension $\geq 2$; see Section 2 for a more precise formulation.)

MSC2000: 14E30.

Keywords: minimal models, Mori fibre spaces. 
Following Shokurov's method and using results of [Birkar et al. 2006], we give a short proof of:

Theorem 1.2. Termination of terminal log flips in dimension $d$ implies Conjecture 1.1 in dimension $d$; more precisely, for a klt pair $(X / Z, B)$ of dimension d one constructs a log minimal model or a Mori fibre space by running the $L M M P / Z$ on $K_{X}+B$ with scaling of a suitable big/Z $\mathbb{R}$-divisor and proving that it terminates.

As in [Shokurov 2006], one immediately derives the following:

Corollary 1.3. Conjecture 1.1 holds in dimension 4.

Note that when $(X / Z, B)$ is effective (for example if it is of nonnegative Kodaira dimension), log minimal models are constructed in [Birkar 2007], using different methods, in dimension $\leq 5$.

\section{Basics}

Let $k$ be an algebraically closed field of characteristic zero. For an $\mathbb{R}$-divisor $D$ on a variety $X$ over $k$, we use $D^{\sim}$ to denote the birational transform of $D$ on a specified birational model of $X$.

Definition 2.1. A pair $(X / Z, B)$ consists of normal quasiprojective varieties $X, Z$ over $k$, an $\mathbb{R}$-divisor $B$ on $X$ with coefficients in $[0,1]$ such that $K_{X}+B$ is $\mathbb{R}$ Cartier, and a projective morphism $X \rightarrow Z$. $(X / Z, B)$ is called $\log$ smooth if $X$ is smooth and Supp $B$ has simple normal crossing singularities.

For a prime divisor $D$ on some birational model of $X$ with a nonempty centre on $X, a(D, X, B)$ denotes the $\log$ discrepancy. $(X / Z, B)$ is terminal in codimension $\geq 2$ if $a(D, X, B)>1$ whenever $D$ is exceptional/X. Log flips preserve this condition but divisorial contractions may not.

Let $(X / Z, B)$ be a klt pair. By a $\log f$ fip/ $Z$ we mean the flip of a $K_{X}+B$ negative extremal flipping contraction $/ Z$. A sequence of $\log$ flips $/ Z$ starting with $(X / Z, B)$ is a sequence $X_{i} \rightarrow X_{i+1} / Z_{i}$ in which $X_{i} \rightarrow Z_{i} \leftarrow X_{i+1}$ is a $K_{X_{i}}+B_{i}$ flip $/ Z$ and $B_{i}$ is the birational transform of $B_{1}$ on $X_{1}$, and $\left(X_{1} / Z, B_{1}\right)=(X / Z, B)$. By termination of terminal log flips in dimension $d$ we mean termination of such a sequence in which $\left(X_{1} / Z, B_{1}\right)$ is a $d$-dimensional klt pair which is terminal in codimension $\geq 2$. Now assume that $G$ is an $\mathbb{R}$-Cartier divisor on $X$. A sequence of $G$-flops $/ Z$ with respect to $(X / Z, B)$ is a sequence $X_{i} \rightarrow X_{i+1} / Z_{i}$ in which $X_{i} \rightarrow Z_{i} \leftarrow X_{i+1}$ is a $G_{i}$-flip $/ Z$ such that $K_{X_{i}}+B_{i} \equiv 0 / Z_{i}$ where $G_{i}$ is the birational transform of $G$ on $X=X_{1}$.

Definition 2.2 ([Birkar 2007, §2]). Let $(X / Z, B)$ be a klt pair, $\left(Y / Z, B_{Y}\right)$ a $\mathbb{Q}$ factorial klt pair, $\phi: X \rightarrow Y / Z$ a birational map such that $\phi^{-1}$ does not contract divisors, and $B_{Y}$ be the birational transform of $B$ (Note that since $X \rightarrow Z$ and 
$Y \rightarrow Z$ are both projective, by the definition of a pair, $X$ and $Y$ have the same image on $Z$ ). Moreover, assume that

$$
a(D, X, B) \leq a\left(D, Y, B_{Y}\right)
$$

for any prime divisor $D$ on birational models of $X$ and assume that the strict inequality holds for any prime divisor $D$ on $X$ which is exceptional $/ Y$.

We say that $\left(Y / Z, B_{Y}\right)$ is a log minimal model of $(X / Z, B)$ if $K_{Y}+B_{Y}$ is nef $/ Z$. On the other hand, we say that $\left(Y / Z, B_{Y}\right)$ is a Morifibre space of $(X / Z, B)$ if there is a $K_{Y}+B_{Y}$-negative extremal contraction $Y \rightarrow Y^{\prime} / Z$ such that $\operatorname{dim} Y^{\prime}<\operatorname{dim} Y$.

Typically, one obtains a log minimal model or a Mori fibre space by a finite sequence of divisorial contractions and log flips.

Remark 2.3. Let $(X / Z, B)$ be a klt pair and $W \rightarrow X$ a log resolution. Let $B_{W}=$ $B^{\sim}+(1-\epsilon) \sum E_{i}$ where $0<\epsilon \ll 1$ and $E_{i}$ are the exceptional/ $X$ divisors on $W$. Remember that $B^{\sim}$ is the birational transform of $B$. If $\left(Y / X, B_{Y}\right)$ is a $\log$ minimal model of $\left(W / X, B_{W}\right)$, which exists by [Birkar et al. 2006], then by the negativity lemma $Y \rightarrow X$ is a small $\mathbb{Q}$-factorialisation of $X$. To find a log minimal model or a Mori fibre space of $(X / Z, B)$, it is enough to find one for $\left(Y / Z, B_{Y}\right)$. So, one could assume that $X$ is $\mathbb{Q}$-factorial by replacing it with $Y$.

We recall a variant of the LMMP with scaling which we use in this paper. Let $(X / Z, B+C)$ be a $\mathbb{Q}$-factorial klt pair such that $K_{X}+B+C$ is nef $/ Z$ and $B, C \geq 0$. By [Birkar 2007, Lemma 2.7], either $K_{X}+B$ is nef $/ Z$ or there is an extremal ray $R / Z$ such that

$$
\left(K_{X}+B\right) \cdot R<0 \text { and }\left(K_{X}+B+\lambda_{1} C\right) \cdot R=0,
$$

where

$$
\lambda_{1}:=\inf \left\{t \geq 0 \mid K_{X}+B+t C \text { is nef } / Z\right\}
$$

and $K_{X}+B+\lambda_{1} C$ is nef $/ Z$. Now assume that $R$ defines a divisorial contraction or a $\log$ flip $X \rightarrow X^{\prime}$. We can consider $\left(X^{\prime} / Z, B^{\prime}+\lambda_{1} C^{\prime}\right)$, where $B^{\prime}+\lambda_{1} C^{\prime}$ is the birational transform of $B+\lambda_{1} C$ and continue the argument. That is, either $K_{X^{\prime}}+B^{\prime}$ is nef $/ Z$ or there is an extremal ray $R^{\prime} / Z$ such that $\left(K_{X^{\prime}}+B^{\prime}\right) \cdot R^{\prime}<0$ and $\left(K_{X^{\prime}}+B^{\prime}+\lambda_{2} C^{\prime}\right) \cdot R^{\prime}=0$, where

$$
\lambda_{2}:=\inf \left\{t \geq 0 \mid K_{X^{\prime}}+B^{\prime}+t C^{\prime} \text { is nef } / Z\right\}
$$

and $K_{X^{\prime}}+B^{\prime}+\lambda_{2} C^{\prime}$ is nef $/ Z$. By continuing this process, we obtain a special kind of LMMP on $K_{X}+B$ which we refer to as the LMMP with scaling of $C$. If it terminates, then we obviously get a log minimal model or a Mori fibre space for $(X / Z, B)$. Note that the required log flips exist by [Birkar et al. 2006]. 


\section{Extremal rays}

In this section, for convenience of the reader, we give the proofs of some results about extremal rays [Shokurov 2006, Corollary 9, Addendum 4]. The norm $\|G\|$ of an $\mathbb{R}$-divisor $G$ denotes the maximum of the absolute value of its coeffecients.

Let $X \rightarrow Z$ be a projective morphism of normal quasiprojective varieties. A curve $\Gamma$ on $X$ is called extremal $/ Z$ if it generates an extremal ray $R / Z$ which defines a contraction $X \rightarrow S / Z$, and if for some ample/Z divisor $H$ we have $H \cdot \Gamma=\min \{H \cdot \Sigma\}$, where $\Sigma$ ranges over curves generating $R$. If $(X / Z, B)$ is divisorial $\log$ terminal $(\mathrm{dlt})$ and $\left(K_{X}+B\right) \cdot R<0$, then by [Kawamata 1991] there is a curve $\Sigma$ generating $R$ such that $\left(K_{X}+B\right) \cdot \Sigma \geq-2 \operatorname{dim} X$. On the other hand, since $\Gamma$ and $\Sigma$ both generate $R$ we have

$$
\frac{\left(K_{X}+B\right) \cdot \Gamma}{H \cdot \Gamma}=\frac{\left(K_{X}+B\right) \cdot \Sigma}{H \cdot \Sigma},
$$

hence

$$
\left(K_{X}+B\right) \cdot \Gamma=\left(K_{X}+B\right) \cdot \Sigma \frac{H \cdot \Gamma}{H \cdot \Sigma} \geq-2 \operatorname{dim} X .
$$

Remark 3.1. Let $(X / Z, B)$ be a $\mathbb{Q}$-factorial klt pair, $F$ be a reduced divisor on $X$ whose support contains that of $B$, and $V$ be the $\mathbb{R}$-vector space of divisors generated by the components of $F$.

(i) By [Shokurov 1992, Property 1.3.2; 1996, First Main Theorem 6.2 and Remark 6.4], the sets

$$
\mathscr{L}=\{\Delta \in V \mid(X / Z, \Delta) \text { is lc }\} \quad \text { and } \mathcal{N}=\left\{\Delta \in \mathscr{L} \mid K_{X}+\Delta \text { is nef } / Z\right\}
$$

are rational polytopes in $V$. Since $B \in \mathscr{L}$, there are rational boundaries $B^{1}, \ldots, B^{r} \in \mathscr{L}$ and nonnegative real numbers $a_{1}, \ldots, a_{r}$ such that $B=$ $\sum a_{j} B^{j}, \sum a_{j}=1$, and each $\left(X / Z, B^{j}\right)$ is klt. In particular, there is $m \in \mathbb{N}$ such that $m\left(K_{X}+B^{j}\right)$ are Cartier, and for any curve $\Gamma$ on $X$ the intersection number $\left(K_{X}+B\right) \cdot \Gamma$ can be written as $\sum a_{j} \frac{n_{j}}{m}$ for certain $n_{1}, \ldots, n_{r} \in \mathbb{Z}$. Moreover, if $\Gamma$ is extremal $/ Z$, then the $n_{j}$ satisfy $n_{j} \geq-2 m \operatorname{dim} X$.

(ii) If $K_{X}+B$ is nef $/ Z$, then $B \in \mathcal{N}$ and so one can choose the $B^{j}$ so that $K_{X}+B^{j}$ are nef $/ Z$.

Lemma 3.2. Let $(X / Z, B)$ be a $\mathbb{Q}$-factorial klt pair. There is a real number $\alpha>0$ such that:

(i) If $\Gamma$ is any extremal curve $/ Z$ and if $\left(K_{X}+B\right) \cdot \Gamma>0$, then $\left(K_{X}+B\right) \cdot \Gamma>\alpha$.

(ii) If $K_{X}+B$ is nef $/ Z$, then for any $\mathbb{R}$-divisor $G$, any sequence $X_{i} \rightarrow X_{i+1} / Z_{i}$ of $G$-flops $/ Z$ with respect to $(X / Z, B)$, and any extremal curve $\Gamma$ on $X_{i}$, if $\left(K_{X_{i}}+B_{i}\right) \cdot \Gamma>0$, then $\left(K_{X_{i}}+B_{i}\right) \cdot \Gamma>\alpha$ where $B_{i}$ is the birational transform of $B$. 
Proof. (i) If $B$ is a $\mathbb{Q}$-divisor, then the statement is trivially true. Let $B^{1}, \ldots, B^{r}$, $a_{1}, \ldots, a_{r}$, and $m$ be as in Remark 3.1(i). Let $\Gamma$ be an extremal curve/Z. Then, $\left(K_{X}+B\right) \cdot \Gamma=\sum a_{j}\left(K_{X}+B^{j}\right) \cdot \Gamma$ and since for each $j$ we have $\left(K_{X}+B^{j}\right) \cdot \Gamma \geq$ $-2 \operatorname{dim} X$, the existence of $\alpha$ is clear for (i).

(ii) By Remark 3.1(ii) we may also assume that $K_{X}+B^{j}$ are nef $/ Z$. Then, the sequence $X_{i} \rightarrow X_{i+1} / Z_{i}$ is also a sequence of flops with respect to each $\left(X / Z, B^{j}\right)$. In particular, $\left(X_{i} / Z, B_{i}^{j}\right)$ is klt and $m\left(K_{X_{i}}+B_{i}^{j}\right)$ is Cartier for any $j, i$ where $B_{i}^{j}$ is the birational transform of $B^{j}$. The rest is as in (i).

Proposition 3.3. Let $(X / Z, B)$ be a $\mathbb{Q}$-factorial klt pair, $F$ a reduced divisor on $X$ whose support contains that of $B$, and $\mathscr{L}$ as in Remark 3.1. There is a rational polytope $\mathscr{K} \subset \mathscr{L}$ of klt boundaries and of maximal dimension containing an open neighborhood of $B$ in $\mathscr{L}$ (with respect to the topology on $\mathscr{L}$ induced by the norm $\|\cdot\|)$ such that

(i) if $\Delta \in \mathscr{K}$ and $\left(K_{X}+\Delta\right) \cdot R<0$ for an extremal ray $R / Z$, then $\left(K_{X}+B\right) \cdot R \leq 0$, and

(ii) if $K_{X}+B$ is nef/Z, $\Delta \in \mathscr{K}$, we have a sequence $X_{i} \rightarrow X_{i+1} / Z_{i}$ of $K_{X}+\Delta-$ flops $/ Z$ with respect to $(X / Z, B)$, and $\left(K_{X_{i}}+\Delta_{i}\right) \cdot R<0$ for an extremal ray $R / Z$ on some $X_{i}$, then $\left(K_{X_{i}}+B_{i}\right) \cdot R=0$, where $\Delta_{i}, B_{i}$ are the birational transforms of $\triangle, B$ respectively.

Proof. (i) Let $\mathcal{M} \subset \mathscr{L}$ be a rational polytope of klt boundaries and of maximal dimension containing an open neighborhood of $B$ in $\mathscr{L}$. If the statement is not true then there is an infinite sequence of $\Delta_{l} \in \mathcal{M}$ and extremal rays $R_{l} / Z$ such that for each $l$ we have

$$
\left(K_{X}+\Delta_{l}\right) \cdot R_{l}<0, \quad\left(K_{X}+B\right) \cdot R_{l}>0,
$$

and $\left\|\Delta_{l}-B\right\|$ converges to 0 . Let $\Omega_{l}$ be the point on the boundary of $\mu$ such that $\Omega_{l}-\Delta_{l}=b_{l}\left(\Delta_{l}-B\right)$ for some real number $b_{l} \geq 0$ and such that $\left\|\Omega_{l}-B\right\|$ is maximal. So, $\Omega_{l}$ is the most far away point in $\mu$ which is on the line determined by $B$ and $\Delta_{l}$, in the direction of $\Delta_{l}$. Since $\left\|\Delta_{l}-B\right\|$ converges to $0, b_{l}$ converges to $+\infty$.

By assumptions, $\left(X / Z, \Omega_{l}\right)$ is klt and if $\Gamma_{l}$ is an extremal curve/ $Z$ generating $R_{l}$, then

$$
\left(\Omega_{l}-\Delta_{l}\right) \cdot \Gamma_{l}=\left(K_{X}+\Omega_{l}\right) \cdot \Gamma_{l}-\left(K_{X}+\Delta_{l}\right) \cdot \Gamma_{l} \geq-2 \operatorname{dim} X .
$$

This is not possible because by Lemma 3.2,

$$
\left(K_{X}+\Delta_{l}\right) \cdot \Gamma_{l}+\left(B-\Delta_{l}\right) \cdot \Gamma_{l}=\left(K_{X}+B\right) \cdot \Gamma_{l}>\alpha,
$$

and by the same arguments $\left(B-\Delta_{l}\right) \cdot \Gamma_{l}$ approaches 0 . 
By definition, the sequence $X_{i} \rightarrow X_{i+1} / Z_{i}$ is a sequence of $K_{X}+\Delta$-flips which are numerically trivial with respect to $K_{X}+B$. Let $\mathscr{K}$ be as in (i). Assume that $R$ is an extremal ray $/ Z$ on $X_{i}$ such that $\left(K_{X_{i}}+\Delta_{i}\right) \cdot R<0$ but $\left(K_{X_{i}}+B_{i}\right) \cdot R>0$. Let $\Gamma$ be an extremal curve $/ Z$ generating $R$. Let $\Omega$ be the point on the boundary of $\mathscr{K}$ which is chosen for $\Delta$ similarly as in (i). By assumptions, $\left(X_{i} / Z, \Delta_{i}\right)$ and $\left(X_{i} / Z, \Omega_{i}\right)$ are klt where $\Omega_{i}$ is the birational transform of $\Omega$. So,

$$
\left(\Omega_{i}-\Delta_{i}\right) \cdot \Gamma=\left(K_{X_{i}}+\Omega_{i}\right) \cdot \Gamma-\left(K_{X_{i}}+\Delta_{i}\right) \cdot \Gamma \geq-2 \operatorname{dim} X .
$$

On the other hand, $\left(K_{X_{i}}+B_{i}\right) \cdot \Gamma>\alpha$ where $\alpha$ is as in Lemma 3.2. By construction, there is some $b \geq 0$ such that $b\left(\Delta_{i}-B_{i}\right)=\Omega_{i}-\Delta_{i}$. Therefore,

$$
\left(K_{X_{i}}+\Delta_{i}\right) \cdot \Gamma=\left(K_{X_{i}}+B_{i}\right) \cdot \Gamma+\left(\Delta_{i}-B_{i}\right) \cdot \Gamma>\alpha-\frac{2 \operatorname{dim} X}{b} .
$$

which is not possible if $b \alpha>2 \operatorname{dim} X$. In other words, if $\Delta$ is close enough to $B$ then the statement of ( $i i$ ) also holds, that is, we only need to shrink $\mathscr{K}$ appropriately.

\section{Proof of the main results}

Proof of Theorem 1.2. Let $(X / Z, B)$ be a klt pair of dimension $d$. By Remark 2.3, we can assume that $X$ is $\mathbb{Q}$-factorial. Let $H \geq 0$ be an $\mathbb{R}$-divisor which is big/ $Z$ so that $K_{X}+B+H$ is klt and nef $/ Z$. Run the LMMP/Z on $K_{X}+B$ with scaling of $H$. If the LMMP terminates, then we get a log minimal model or a Mori fibre space. Suppose that we get an infinite sequence $X_{i} \rightarrow X_{i+1} / Z_{i}$ of $\log$ flips $/ Z$, where we may also assume that $\left(X_{1} / Z, B_{1}\right)=(X / Z, B)$.

Let $\lambda_{i}$ be the threshold on $X_{i}$ determined by the LMMP with scaling as explained in Section 2. So, $K_{X_{i}}+B_{i}+\lambda_{i} H_{i}$ is nef $/ Z$,

$$
\left(K_{X_{i}}+B_{i}\right) \cdot R_{i}<0 \quad \text { and } \quad\left(K_{X_{i}}+B_{i}+\lambda_{i} H_{i}\right) \cdot R_{i}=0,
$$

where $B_{i}$ and $H_{i}$ are the birational transforms of $B$ and $H$ and, $R_{i}$ is the extremal ray which defines the flipping contraction $X_{i} \rightarrow Z_{i}$. Obviously, $\lambda_{i} \geq \lambda_{i+1}$.

Put $\lambda=\lim _{i \rightarrow \infty} \lambda_{i}$. If the limit is attained, that is, $\lambda=\lambda_{i}$ for some $i$, then the sequence terminates by Corollary 1.4.2 of [Birkar et al. 2006]. So, we assume that the limit is not attained. Actually, if $\lambda>0$, again [Birkar et al. 2006] implies that the sequence terminates. However, we do not need to use [Birkar et al. 2006] in this case. In fact, by replacing $B_{i}$ with $B_{i}+\lambda H_{i}$, we can assume that $\lambda=0$ hence $\lim _{i \rightarrow \infty} \lambda_{i}=0$.

Put $\Lambda_{i}:=B_{i}+\lambda_{i} H_{i}$. Since we are assuming that terminal log flips terminate, or, alternatively, by Corollary 1.4.3 of [Birkar et al. 2006], we can construct a terminal (in codimension $\geq 2$ ) crepant model $\left(Y_{i} / Z, \Theta_{i}\right)$ of $\left(X_{i} / Z, \Lambda_{i}\right)$. A slight modification of the argument in Remark 2.3 would do this. Note that we can assume 
that all the $Y_{i}$ are isomorphic to $Y_{1}$ in codimension one, perhaps after truncating the sequence. Let $\Delta_{1}=\lim _{i \rightarrow \infty} \Theta_{i}^{\sim}$ on $Y_{1}$ and let $\Delta_{i}$ be its birational transform on $Y_{i}$. The limit is obtained componentwise.

Since $H_{i}$ is big/ $Z$ and $K_{X_{i}}+\Lambda_{i}$ is klt and nef $/ Z, K_{X_{i}}+\Lambda_{i}$ and $K_{Y_{i}}+\Theta_{i}$ are semiample $/ Z$ by the base point freeness theorem for $\mathbb{R}$-divisors. Thus, $K_{Y_{i}}+\Delta_{i}$ is a limit of movable $/ Z$ divisors which in particular means that it is pseudo-effective $/ Z$. Note that if $K_{Y_{i}}+\Delta_{i}$ is not pseudo-effective/Z, we get a contradiction by Corollary 1.3.2 of [Birkar et al. 2006].

Now run the LMMP $/ Z$ on $K_{Y_{1}}+\Delta_{1}$. No divisor will be contracted again because $K_{Y_{1}}+\Delta_{1}$ is a limit of movable $/ Z$ divisors. Since $K_{Y_{1}}+\Delta_{1}$ is terminal in codimension $\geq 2$, by assumptions, the LMMP terminates with a log minimal model $(W / Z, \Delta)$. By construction, $\Delta$ on $W$ is the birational transform of $\Delta_{1}$ on $Y_{1}$, and $G_{i}:=\Theta_{i}^{\sim}-\Delta$ on $W$ satisfies $\lim _{i \rightarrow \infty} G_{i}=0$.

By Proposition 3.3, for each $G_{i}$ with $i \gg 0$, we can run the LMMP/Z on $K_{W}+$ $\Delta+G_{i}$ which will be a sequence of $G_{i}$-flops, that is, $K+\Delta$ would be numerically zero on all the extremal rays contracted in the process. No divisor will be contracted because $K_{W}+\Delta+G_{i}$ is movable/Z. The LMMP ends up with a log minimal model $\left(W_{i} / Z, \Omega_{i}\right)$. Here, $\Omega_{i}$ is the birational transform of $\Delta+G_{i}$ and so of $\Theta_{i}$. Let $S_{i}$ be the lc model of $\left(W_{i} / Z, \Omega_{i}\right)$ which is the same as the lc model of $\left(Y_{i} / Z, \Theta_{i}\right)$ and that of $\left(X_{i} / Z, \Lambda_{i}\right)$ because $K_{W_{i}}+\Omega_{i}$ and $K_{Y_{i}}+\Theta_{i}$ are nef $/ Z$ with $W_{i}$ and $Y_{i}$ being isomorphic in codimension one, and $K_{Y_{i}}+\Theta_{i}$ is the pullback of $K_{X_{i}}+\Lambda_{i}$. Also note that since $K_{X_{i}}+B_{i}$ is pseudoeffective $/ Z, K_{X_{i}}+\Lambda_{i}$ is big/Z; hence $S_{i}$ is birational to $X_{i}$.

By construction, $K_{W_{i}}+\Delta^{\sim}$ is nef/Z and it turns out that $K_{W_{i}}+\Delta^{\sim} \sim_{\mathbb{R}} 0 / S_{i}$. Suppose that this is not the case. Then, $K_{W_{i}}+\Delta^{\sim}$ is not numerically zero/ $S_{i}$ hence there is some curve $C / S_{i}$ such that $\left(K_{W_{i}}+\Delta^{\sim}+G_{i}^{\sim}\right) \cdot C=0$ but $\left(K_{W_{i}}+\Delta^{\sim}\right) \cdot C>0$ which implies that $G_{i}^{\sim} \cdot C<0$. Hence, there is a $K_{W_{i}}+\Delta^{\sim}+(1+\tau) G_{i}^{\sim}$-negative extremal ray $R / S_{i}$ for any $\tau>0$. This contradicts Proposition 3.3 because we must have

$$
\left(K_{W_{i}}+\Delta^{\sim}+G_{i}^{\sim}\right) \cdot R=\left(K_{W_{i}}+\Delta^{\sim}\right) \cdot R=0 .
$$

Therefore, $K_{W_{i}}+\Delta^{\sim} \sim_{\mathbb{R}} 0 / S_{i}$. Now $K_{X_{i}}+\Lambda_{i} \sim_{\mathbb{R}} 0 / Z_{i}$ implies that $Z_{i}$ is over $S_{i}$ and so $K_{Y_{i}}+\Delta_{i} \sim_{\mathbb{R}} 0 / S_{i}$. On the other hand, $K_{X_{i}}+B_{i}$ is the pushdown of $K_{Y_{i}}+\Delta_{i}$; hence $K_{X_{i}}+B_{i} \sim_{\mathbb{R}} 0 / S_{i}$. Thus, $K_{X_{i}}+B_{i} \sim_{\mathbb{R}} 0 / Z_{i}$ and this contradicts the fact that $X_{i} \rightarrow Z_{i}$ is a $K_{X_{i}}+B_{i}$-flipping contraction. So, the sequence of flips terminates and this completes the proof.

Proof of Corollary 1.3. Since terminal log flips terminate in dimension 4 by [Fujino 2004; Shokurov 2004] (see also [Alexeev et al. 2007]), the result follows from Theorem 1.2. 


\section{Acknowledgements}

I thank V. V. Shokurov and Y. Kawamata for their comments and suggestions, which improved this paper.

\section{References}

[Alexeev et al. 2007] V. Alexeev, C. Hacon, and Y. Kawamata, "Termination of (many) 4-dimensional log flips”, Invent. Math. 168:2 (2007), 433-448. MR 2008f:14028 Zbl 1118.14017

[Birkar 2007] C. Birkar, "On existence of log minimal models”, preprint, 2007. arXiv 0706.1792

[Birkar et al. 2006] C. Birkar, P. Cascini, C. Hacon, and J. McKernan, "Existence of minimal models for varieties of log general type”, preprint, 2006. arXiv math/0610203

[Fujino 2004] O. Fujino, "Termination of 4-fold canonical flips", Publ. Res. Inst. Math. Sci. 40:1 (2004), 231-237. Addendum: 41:1 (2005), 251-257. MR 2004k:14020 Zbl 1069.14017

[Kawamata 1991] Y. Kawamata, "On the length of an extremal rational curve", Invent. Math. 105:3 (1991), 609-611. MR 92m:14026 Zbl 0751.14007

[Shokurov 1992] V. V. Shokurov, “Three-dimensional log flips”, Izv. Ross. Akad. Nauk Ser. Mat. 56:1 (1992), 105-203. In Russian; translated in Russian Acad. Sci. Izv. Math. 40:1 (1993), 95-202. MR 93j:14012 Zbl 0785.14023

[Shokurov 1996] V. V. Shokurov, “3-fold log models”, Journal of Math. Sci. 81:3 (1996), 26672699. MR 97i:14015 Zbl 0873.14014

[Shokurov 2004] V. V. Shokurov, "Letters of a bi-rationalist, V: Minimal log discrepancies and termination of log flips", pp. 328-351 in Algebr. Geom. Metody, Svyazi i Prilozh., Tr. Mat. Inst. Steklova 246, 2004. In Russian; translated in Proc. Stekl. Inst. Mathematics 246, (2004), 315-336. MR 2006b:14019 Zbl 1107.14012

[Shokurov 2006] V. V. Shokurov, "Letters of a bi-rationalist, VII: Ordered termination", preprint, 2006. arXiv math/0607822

Communicated by Yujiro Kawamata

Received 2009-03-12 Revised 2009-09-07 Accepted 2009-10-06

c.birkar@dpmms.cam.ac.uk DPMMS, Centre for Mathematical Sciences, Cambridge University, Wilberforce Road, Cambridge, CB3 OWB, United Kingdom 


\section{Algebra \& Number Theory}

www.jant.org

\section{EDITORS}

\section{MANAGING EDITOR}

Bjorn Poonen

Massachusetts Institute of Technology

Cambridge, USA

\author{
EDITORIAL BOARD CHAIR \\ David Eisenbud \\ University of California \\ Berkeley, USA
}

\section{BOARD OF EDITORS}

\section{Georgia Benkart}

Dave Benson

Richard E. Borcherds

John H. Coates

J-L. Colliot-Thélène

Brian D. Conrad

Hélène Esnault

Hubert Flenner

Edward Frenkel

Andrew Granville

Joseph Gubeladze

Ehud Hrushovski

Craig Huneke

Mikhail Kapranov

Yujiro Kawamata

János Kollár

Hendrik W. Lenstra

Yuri Manin

Barry Mazur
University of Wisconsin, Madison, USA

University of Aberdeen, Scotland

University of California, Berkeley, USA

University of Cambridge, UK

CNRS, Université Paris-Sud, France

University of Michigan, USA

Universität Duisburg-Essen, Germany

Ruhr-Universität, Germany

University of California, Berkeley, USA

Université de Montréal, Canada

San Francisco State University, USA

Hebrew University, Israel

University of Kansas, USA

Yale University, USA

University of Tokyo, Japan

Princeton University, USA

Universiteit Leiden, The Netherlands

Northwestern University, USA

Harvard University, USA
Susan Montgomery

Shigefumi Mori

Andrei Okounkov

Raman Parimala

Victor Reiner

Karl Rubin

Peter Sarnak

Michael Singer

Ronald Solomon

Vasudevan Srinivas

J. Toby Stafford

Bernd Sturmfels

Richard Taylor

Ravi Vakil

Michel van den Bergh

Marie-France Vignéras

Kei-Ichi Watanabe

Andrei Zelevinsky

Efim Zelmanov
University of Southern California, USA

RIMS, Kyoto University, Japan

Princeton University, USA

Emory University, USA

University of Minnesota, USA

University of California, Irvine, USA

Princeton University, USA

North Carolina State University, USA

Ohio State University, USA

Tata Inst. of Fund. Research, India

University of Michigan, USA

University of California, Berkeley, USA

Harvard University, USA

Stanford University, USA

Hasselt University, Belgium

Université Paris VII, France

Nihon University, Japan

Northeastern University, USA

University of California, San Diego, USA

\section{PRODUCTION}

ant@mathscipub.org

Paulo Ney de Souza, Production Manager

Silvio Levy, Senior Production Editor

See inside back cover or www.jant.org for submission instructions.

The subscription price for 2009 is US \$140/year for the electronic version, and \$200/year (+\$35 shipping outside the US) for print and electronic. Subscriptions, requests for back issues from the last three years and changes of subscribers address should be sent to Mathematical Sciences Publishers, Department of Mathematics, University of California, Berkeley, CA 94720-3840, USA.

Algebra \& Number Theory (ISSN 1937-0652) at Mathematical Sciences Publishers, Department of Mathematics, University of California, Berkeley, CA 94720-3840 is published continuously online. Periodical rate postage paid at Berkeley, CA 94704, and additional mailing offices.

ANT peer-review and production is managed by EditFLOw ${ }^{\mathrm{TM}}$ from Mathematical Sciences Publishers.

PUBLISHED BY

mathematical sciences publishers

http://www.mathscipub.org

A NON-PROFIT CORPORATION

Typeset in LATEX

Copyright $\odot 2009$ by Mathematical Sciences Publishers 


\section{Algebra \& Number Theory}

Volume $3 \quad$ No. $8 \quad 2009$

On coproducts in varieties, quasivarieties and prevarieties

GEORGE M. BERGMAN

Exponential sums nondegenerate relative to a lattice

Alan AdolphSON and STEVEN SPERBER

F-adjunction

KARL SCHWEDE

Log minimal models according to Shokurov

CAUCHER BIRKAR

Shlomo GELAKI, DEEPAK NAIDU and DMITRI NIKSHYCH 